\section{How COVID (Pandemics) Impacted Training of Medical Students, Trainees, and Resi- dents?}

Sir,

COVID-19 definitely surprised all the world by transforming from being a disease confined in a city to being a country epidemic; and finally getting a pandemic status. This new pandemic brought a myriad of challenges. Hospitals changed the way of working and adopted new strategies. They increased their bed strength, upgraded wards with ICU capabilities, postponed elective surgeries and gave new roles to healthcare providers. This all has impacted medical education and training, while there have been daily changes in the strategy to deal with the pandemic and also have been significant changes in the teaching practices. ${ }^{1} \mathrm{~A}$ total halt of educational services in the phase of lockdown led to a new era of e-learning with the use of various internet meeting platforms. While these come with an advantage of easy availability and a wide reach, but there is a limit to how effective they can be in terms of imparting clinical knowledge. Surely, there can be vivid presentations addressing the theoretical part of the subject and 3-D animations on clinical examination with the help of newer pictographic tools available to us. But, can this replace the old school hands-on teaching? Will the newer generation of medical students be able to apply these virtually learned skills when it comes to actually dealing with the real human body? These are a few challenging questions that need to be answered as we adapt ourselves with these newer methods of teaching.

Initially, the students faced a great deal of uncertainty during the lockdown as to whether the classes are going to resume or not. Those students who were supposed to have their rotations suffered the most as all forms of patient contact was removed and most of them eventually received full credits for clinical rotations. This is concerning as to what will the students do when they will actually face a specific type of patient which they have never had a direct clinical contact with. There was no clear-cut information regarding the pattern and time of the examinations. Some lost the precious time of their training, while others missed the deadlines of their competitive entrance examinations. And to top all of that, residents had duties in the COVID wards, ICUs and screening centres. Being struck in a cycle of duties and self-quarantine with an added fear of contracting the disease, many residents suffered from depression and anxiety. Poor mental health certainly had a worse effect on the studies and training of such residents. ${ }^{2}$

E-learning platforms came as a blessing in these difficult times and gradually the teaching resumed. We struggled in the starting while using such online platforms as it was new, more for the teachers than for the students in terms of the technological aspects. ${ }^{3}$ But with time, even the clinical postings were being organised online with the help of pictorial presentations of techniques. These platforms definitely helped us in giving a normal routine to a student who was initially clueless and skeptical about future. The online applications provided very useful interface of giving daily assignments to the students and keep them occupied. Palan et al. reported that establishing and conducting virtual learning platforms and journal clubs help to optimise resident time spent away from the hospital. ${ }^{4}$ They found a number of unique strengths relative to a traditional inperson learning format, such as the ability to revisit the digitally recorded session for clarification or continued learning, ease of access to both clinical materials and key articles, and the ability to connect individuals of different geographic regions. Even the examinations were conducted in many medical institutions with the help of these aids. ${ }^{3}$

With the wide spectrum of disease (asymptomatic to critically ill), it was difficult to manage all patients in smaller centres and for that tertiary care centres had to be involved. While some of these are functioning exclusively as COVID hospitals, the rest are also dealing with a significant number of cases leading to a crunch in the available resources including hospital beds, equipment, operation theatre time and medical personnel. As a result, there is a decrease in the number of in-patient admissions for diagnostic workups and elective procedures, and mostly being done, are emergency procedures. All this is jeopardising the training of the residents in these institutions. These centres where hundreds of residents come to pursue their residency with the dream of being trained in their respective subjects by the best, are somewhere helpless in this current scenario as far as the medical education is concerned.

You can watch a procedure online for many times and still be surprised when you actually have to do it. Human body is full of surprises and medical training is all about practice and an allround personality development of a trainee where he or she knows how to go about in the most difficult of scenarios. "Patients are the best teachers" is an age-old saying in medical training and with the current norms of social distancing, it seems that this phrase is losing its meaning. There is no doubt that the new digital tools bring the teacher and student just a click away from each other, but the medium of teaching, which is the patient himself, is missing. An important aspect of medical education is history taking, where a trainee learns the basic approach, how to talk to the patient, how to gain his or her confidence, and how to take out the relevant information necessary to reach a diagnosis. It is indeed an important skill, which is being neglected in the current times of e-learning.

What is the long-term solution?

We should try to focus on establishing an environment which is safe with observation of all the social distancing norms and still allows us to teach our residents on the patients themselves. E- 
learning can be used as an add-on to sharpen the knowledge and basic skill of our residents with clinical scenario-based presentations, which make the residents question their mind that what should be the next step for such a case. By being totally reliant on the online platform, we are actually distancing the students from the patients and a time will come when we see an army of doctors who knows how to help their patients on pen and paper, but don't actually know how to perform it in real. As demand and pressure on residents keep increasing, we should keep medical education and training as our top most priority.

\section{CONFLICT OF INTEREST:}

The authors declared no conflict of interest.

\section{AUTHORS' CONTRIBUTION:}

SR, AN, PKS: Conception of the work; interpretation of data for the work, drafting and reviewing the manuscript, final approval of the version, accountable for work.

\section{REFERENCES}

1. Kogan M, Klein SP, Hannon CP, Hannon M. Orthopaedic education during the COVID-19 pandemic. J Am Acad Orthop Surg 2020; doi: 10.5435/JAAOS-D-20-00292.

2. Theoret $C$, Ming $X$. Our education, our concerns: The impact on medical student education of COVID-19. Med Educ 2020; 54(7):591-2. doi: 10.1111/medu.14181.
3. Huynh R. The role of E-learning in medical education. Acad Med 2017; 92(4):430. doi: 10.1097/ACM.000000000000 1596.

4. Palan J, Roberts V, Bloch B, Kulkarni A, Bhowal B, Dias J. The use of a virtual learning environment in promoting virtual journal clubs and case-based discussions in trauma and orthopaedic postgraduate medical education: The Leicester experience. J Bone Joint Surg Br 2012; 94(9): 1170-5. doi: 10.1302/0301-620X.94B9.28780.

Seema Rawat ${ }^{1}$, Pankaj Kumar Sharma ${ }^{2}$ and Amit Narang ${ }^{3}$

${ }^{1}$ Department of Gynecology and Obstetrics, UCMS, Govt Medical Collage, New Delhi, India

${ }^{2}$ Department of Orthopedics, All India Institute of Medical Sciences, Bathinda, India

${ }^{3}$ Department of Orthopedics, Lady Hardinge Medical College, New Delhi, India

Correspondence to: Dr. Pankaj Kumar Sharma, Department of Orthopedics, All India Institute of Medical Sciences, Bathinda, India

E-mail:dr.pankajkristwal@gmail.com

Received: May 04, 2021; Revised: June 07, 2021;

Accepted: June 23, 2021

DOI: https://doi.org/10.29271/jcpsp.2021.JCPSPCR.CR147 